\title{
Supporting Information for Breathable, Stimuli-Responsive, and Self-Sealing Chemical Barrier Material based on Selectively Superabsorbing Polymer
}

Kenneth C. Manning, ${ }^{1}$ Praveen Kotagama, ${ }^{1}$ Timothy P. Burgin,${ }^{2,3^{*}}$ and Konrad Rykaczewski ${ }^{*}$

1. School for Engineering of Matter, Transport and Energy, Arizona State University, Tempe, AZ, 85287, USA

2. Joint Research and Development Inc., 50 Tech Parkway, Stafford, VA 22556, USA

3. School of Molecular Sciences, Arizona State University, Tempe, AZ, 85287, USA

*corresponding author emails: konradr@asu.edu and tburgin@jrad.us

\section{SAP particle size distribution}

Using optical microscopy, we estimated the effective diameter of the SAP particles. While the

particles show non-spherical geometry (typical for ground materials) we used the cross-sectional area, measured using ImageJ, to estimate the effective diameter for the equivalent area sphere. These diameters were then averaged by number to provide an estimate of the average particle size $(44 \pm 19 \mu \mathrm{m})$. 


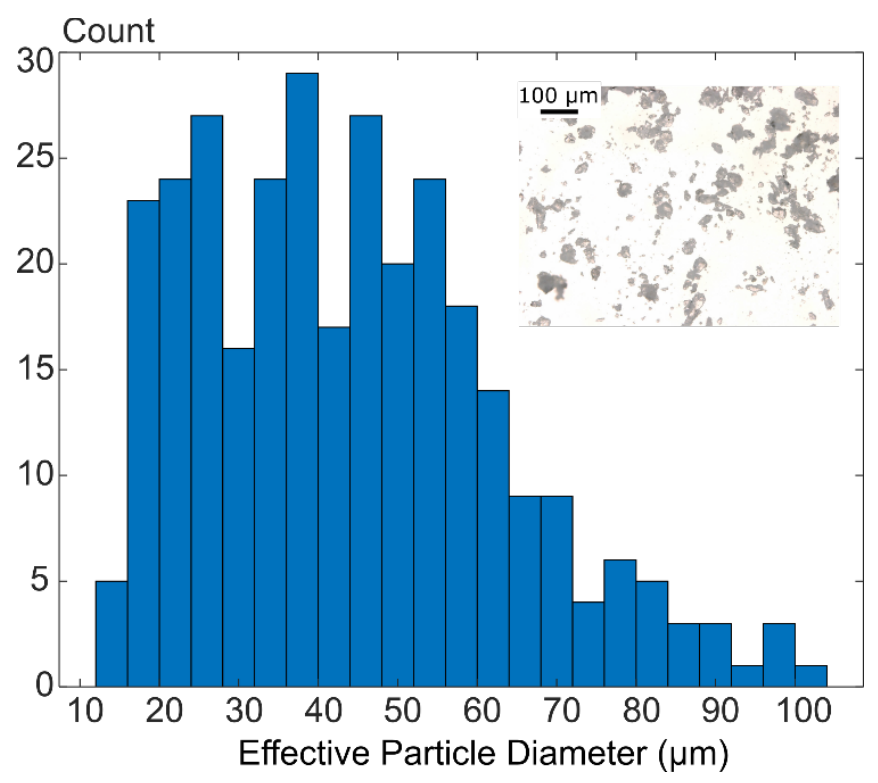

Figure S1. The effective diameters of the super absorbent polymer particles. Inset shows a representative image taken from the optical microscope.

\section{SAP swelling rates}

We tested polymer swelling on the bead of SAP depositing a $2 \mu \mathrm{L}$ droplet of $o$-xylene on a very thinly coated mesh. The results were captured using a Zeiss AxioVision microscope as a time lapse. 

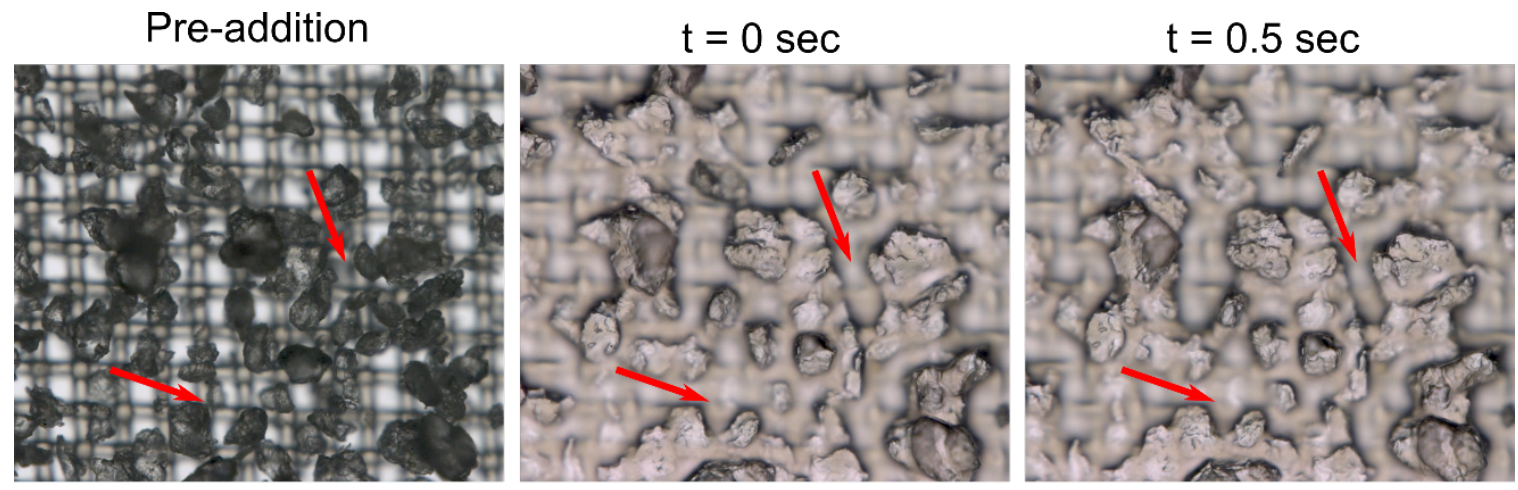

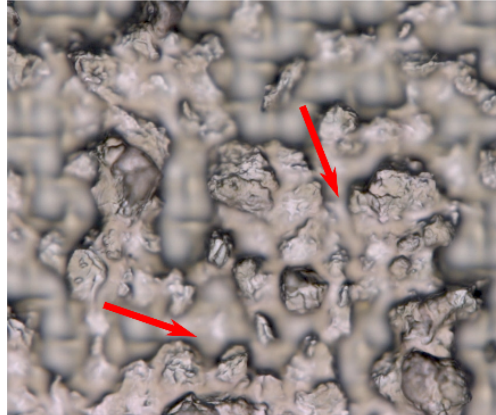

$\mathrm{t}=1 \mathrm{sec}$

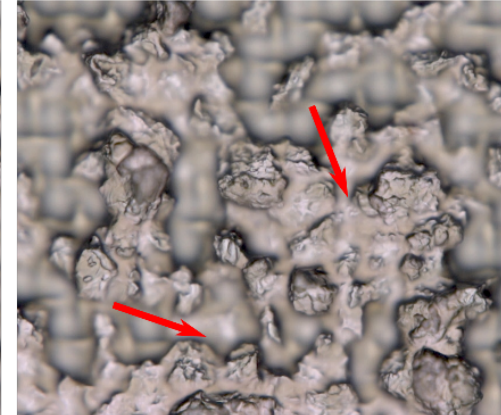

$t=1.5 \mathrm{sec}$

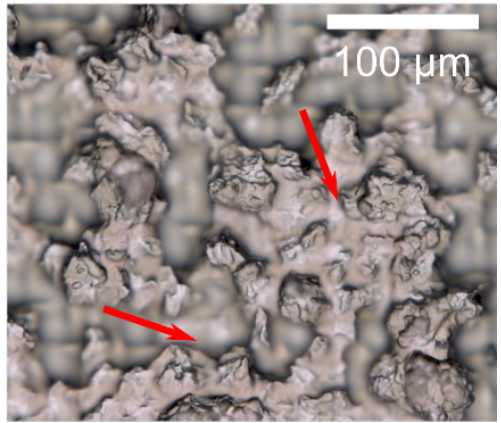

$\mathrm{t}=2 \mathrm{sec}$

Figure S2. Time lapse of polymer swelling in o-xylene dyed with oil red. The image in $t=0$ seconds is split between the pre (left) and post (right) exposure at the same timepoint for comparative purposes. 